\section{SISTEMAS DISTRITUALES DE INNOVACIÓN}

\author{
Daniel Gabaldón Estevan \\ Departamento de Sociología y Antropología Social. \\ Universitat de València \\ daniel.gabaldon@uv.es \\ INGENIO-Instituto de Gestión de la Innovación \\ $y$ del Conocimiento (CSIC-UPV). \\ Universidad Politécnica de Valencia \\ Ignacio Fernández de Lucio \\ INGENIO-Instituto de Gestión de la Innovación \\ $y$ del Conocimiento (CSIC-UPV). \\ Universidad Politécnica de Valencia \\ ifernand@ingenio.upv.es \\ Francesc Xavier Molina Morales \\ Departamento de Administración de Empresas y Marketing. \\ Universitat Jaume I \\ molina@emp.uji.es
}

\begin{abstract}
The innovation system approach shows growing presence in the debate about the determinants of innovation, and has relevant implications on innovation policy. This approach identifies the agents and its connexions in different contexts such as territories, sectors or technologies allowing an improved understanding on how innovation processes function. However, often that boundary may not be the appropriate unit of analysis for studying the most significant relationships regarding innovation in certain contexts such as industrial districts. The industrial district approach, on the other hand, offers the appropriate unit of analysis for studying the relevant relations. However, for this approach innovation has not been of central focus. We therefore propose the Distritual Innovation System that merges the innovation system approach, where learning by interacting is central, and the main elements of the industrial district concept. This new conceptual proposal draws various conclusions on and has implications for the debate on the processes of innovation.
\end{abstract}

KEY WORDS: Innovation; innovation system; sectoral innovation system; industrial district.

\section{INTRODUCCIÓN}

En las últimas décadas la innovación se ha convertido en un tema de estudio preferente para diversas disciplinas académicas. Asimismo, el estudio de la innovación y los diversos aspectos de los procesos que llevan a su generación, desarrollo y difusión han interesado de forma particular a las empresas y a los responsables políticos. La

\section{DISTRITUAL INNOVATION SYSTEMS}

RESUMEN: El concepto de sistema de innovación ha tenido un protagonismo creciente en el debate sobre los determinantes de la innovación y sus implicaciones en las políticas de innovación. El sistema de innovación identifica los agentes y sus interrelaciones en diferentes contextos: territorios, sectores o tecnologias y ha permitido un avance significativo en la comprensión de cómo funcionan los procesos innovadores. Sin embargo, frecuentemente el acotamiento puede no resultar el espacio en el que se desarrollan las relaciones más significativas para explicar los procesos de innovación. En nuestra propuesta, el Sistema Distritual de Innovación, tratamos de integrar la perspectiva sistémica y de aprendizaje a través de las interacciones provenientes de los sistemas de innovación con el concepto de distrito industrial, concepto ya consolidado que, si bien no ha considerado la innovación como un elemento clave, si propone una delimitación del ámbito relacional que consideramos apropiada para el estudio de la innovación. Esta propuesta conceptual sugiere una serie de conclusiones e implicaciones relevantes en este debate sobre los procesos de innovación.

PALABRAS CLAVE: Innovación; sistema de innovación; sistema sectorial de innovación; distrito industrial.

innovación también ha sido considerada como un objetivo de investigación de las más diversas disciplinas, y cada vez más estos estudios se están constituyendo por sí mismos como un campo de investigación distintivo.

La innovación ha sido y es analizada desde diversas perspectivas y enfoques disciplinarios, poniendo cada uno de ellos el énfasis en aspectos particulares del fenómeno. 
Aunque en los últimos años se ha producido un gran avance en el entendimiento de cómo se producen y qué determina las innovaciones, es habitual encontrar una falta de integración teórica de perspectivas a la hora de explicar el funcionamiento de mecanismos considerados claves en los procesos innovadores. Suele asumirse que la innovación no es un proceso individual que las empresas puedan desarrollar de forma aislada, como el denominado modelo lineal sugiere (Asheim e Isaksen, 2001). Por el contario, existe un amplio acuerdo de que la innovación depende, en gran medida, del contexto económico y social en el que la empresa opera. Partiendo de esta premisa, nuestro trabajo pretende contribuir al debate sobre la eficacia y utilidad de diversos desarrollos conceptuales que tratan de capturar de qué manera interactúan los agentes y actores de un contexto territorial determinado.

El debate sobre los determinantes de la innovación y sus implicaciones en las políticas de innovación ha dado lugar a un número considerable de trabajos y propuestas. Con el fin de simplificar y hacer más comprensible nuestra propia propuesta hemos tomado como referencia el concepto de sistema de innovación. El concepto de sistema de innovación (Freeman, 1987; Nelson y Rosenberg, 1993; Lundvall, 1992; Edquist, 1997) aporta una visión sistémica de la estructura y las interacciones de los diversos niveles y elementos que intervienen en el proceso innovador. Este concepto se ha aplicado a diversos ámbitos (Oinas y Malecki, 2002) y ha generado una extensa literatura, convirtiéndose en un instrumento básico de análisis del proceso de innovación en contextos territoriales.

Este concepto descansa en una concepción interactiva de los procesos de innovación. La innovación pasa a ser considerada un proceso colectivo y dinámico, llevado a cabo por la empresa a través de una relación activa con otros agentes e instituciones (científicas, industriales o gubernamentales) alejándose así del denominado modelo lineal de innovación. El principal mérito de esta propuesta radica en situar la innovación en el centro del análisis. El entramado de instituciones y organizaciones es evaluado en función de su contribución a la generación, desarrollo y difusión de la innovación. En este sentido, el concepto de sistema de innovación es un instrumento útil para el diagnóstico de la capacidad de innovación de naciones, regiones o sectores, ya que permite visualizar y medir las posibles deficiencias de un sistema determinado.
Reconocemos que la contribución del concepto de sistema de innovación y el posterior desarrollo tanto teórico como empírico ha permitido un avance significativo en la comprensión de cómo funcionan los procesos innovadores. Este enfoque no sólo afianza aún más la visión de un cambio tecnológico "desincorporado", basado en la creación y difusión de conocimientos, sino que además destaca el valor de las interacciones entre las empresas y las diferentes instituciones sociales como factor determinante de la innovación. La empresa no innova sola, sino que se encuentra inmersa en un espacio socioeconómico y cultural determinado en el que están presentes otras instituciones con las que puede interrelacionarse para llevar a cabo sus innovaciones. Es por esto que, en nuestra opinión, cuando se pretende aplicar este modelo a determinadas realidades, las unidades de análisis existentes (nacional, regional o sectorial) resultan poco eficaces para el análisis, ya que el sistema de innovación identifica los agentes o participantes a partir de un espacio político o institucional que no siempre coincide con el espacio en el que se producen las relaciones e interacciones más significativas para explicar los procesos de innovación.

Es a partir de esta posible debilidad del sistema de innovación, que hemos planteado la propuesta del Sistema Distritual de Innovación. En este sentido, y como la propia denominación sugiere, hemos tratado de integrar el concepto de distrito industrial marshalliano (DIM) con el de sistema de innovación. El distrito industrial es un concepto ya consolidado que, aunque inicialmente no ha considerado la innovación como un elemento central (Becattini, 1990), sí que propone una delimitación del ámbito relacional que, en nuestra opinión, puede aportar una contribución significativa al análisis del fenómeno de la innovación. Además, el patrón de relaciones que sugiere el distrito propicia la generación y transmisión de un conocimiento tácito y contextual que se produce en el ámbito de las empresas, más adaptado a las estrategias de explotación y a las innovaciones incrementales. Este patrón completa las innovaciones tecnológicas provenientes del conocimiento explícito que se producen esencialmente en las universidades y centros de investigación y que es más propio de los sistemas de innovación.

De las propuestas de sistemas de innovación existentes, interesa particularmente la perspectiva sectorial desarroIlada en Breschi y Malerba (1997) y Malerba (2002), que 
destaca por su utilización de la noción de sector, como ámbito de delimitación del análisis y se interesa por aquellas empresas, agentes e instituciones que se vinculan con la actividad sectorial independientemente de su ubicación o del tipo de tecnología que utilizan. Si bien el concepto de sistema sectorial se vincula con algunos aspectos de la perspectiva del análisis económico industrial tradicional (como la identificación de los sectores en base a sus productos y demanda, y el énfasis en las tecnologías básicas), se diferencia de éste en diversos aspectos (Malerba, 1999). Por un lado, acentúa la importancia de la base de conocimiento en los procesos de aprendizaje de los agentes, la heterogeneidad de éstos, las instituciones sectoriales y organizaciones distintas a las empresas, la importancia de las complementariedades dinámicas y su énfasis en los procesos de cambio y las dinámicas del sistema a través de procesos de co-evolución. Su análisis se deriva por tanto de los estudios evolucionistas y de sistemas de innovación, siendo su principal diferencia con estos últimos la de centrar su análisis en los sectores y no en las tecnologías o territorios.

Así, la propuesta teórica que vamos a desarrollar parte, por un lado, de que la perspectiva analítica del Sistema Sectorial de Innovación (SSI) ofrece un corpus teórico-metodológico que permite abordar el estudio de los procesos de innovación de una manera más sistemática y estructurada de lo que puede ofrecer el análisis de los DIM. Pero también que, por otro lado, la teoría de los DIM, si bien ofrece un acotamiento espacial ciertamente más preciso para el caso que nos ocupa, no ha tenido como objetivo principal el estudio de la innovación y, aunque varios de sus postulados, como ahora veremos, puedan efectivamente contribuir a comprender este fenómeno en tales ámbitos, su complementación con la perspectiva sistémica la hace más fructífera. Una revisión de ambas literaturas, que de hecho comparten posturas comunes respecto del fenómeno de la innovación, provee de un conjunto de ideas que son útiles para nuestra investigación.

Es por esto que frente al estudio de la innovación desde la perspectiva exclusivamente enmarcada en la teoría SSI, y aprovechando la contribución a su estudio desde la perspectiva DIM, en este trabajo proponemos un concepto sintético, bajo la rúbrica sistema distritual de innovación (SDI), que pretende incorporar conceptos e ideas de ambas propuestas. En este contexto, nuestro trabajado se estruc- tura como sigue: en primer lugar planteamos el marco teórico de referencia, con la descripción de los conceptos de sistema de innovación y de distrito industrial, para después formular la propuesta de integración. Finalmente, en las conclusiones sugerimos las posibles ampliaciones de esta integración.

\section{Elementos desde la perspectiva del sistema SECTORIAL DE INNOVACIÓN}

Malerba (2004) considera que la innovación en un sector determinado se ve afectada por tres factores principales: (1) el conocimiento y la tecnología, (2) los actores y las redes, y (3) las instituciones. Haciéndose eco de la literatura evolucionista, desde la perspectiva sectorial se considera que el conocimiento está en la base del cambio tecnológico aunque, al mismo tiempo, es altamente idiosincrático al nivel de la empresa y no se difunde de modo libre y automático entre empresas, sino que se reabsorbe por éstas a través de las diferentes habilidades que han acumulado a lo largo del tiempo. En sectores en los que las innovaciones son rápidas la frontera del conocimiento y la tecnología es muy cambiante. Los nexos y complementariedades entre artefactos y actividades también son relevantes para establecer los límites de un sistema sectorial. Las complementariedades dinámicas toman en consideración las interdependencias y retroalimentaciones tanto al nivel de la demanda como del nivel productivo, y son la principal fuente de transformación y crecimiento de los sistemas sectoriales, pudiendo iniciar ciclos virtuosos de innovación y cambio.

Por su parte, los actores o agentes pueden ser individuos u organizaciones, y estas últimas a su vez pueden ser, bien empresas, universidades, organizaciones financieras, sindicatos, etc., bien sub-organizaciones de éstas como departamentos de I+D, o bien grupos de organizaciones como las asociaciones empresariales. En los agentes se incluyen, junto a las empresas y a las organizaciones no empresariales, tanto a los proveedores como a los clientes y, según el tipo de industria, cada uno de éstos puede jugar un papel más o menos fundamental. Estos agentes heterogéneos, que se caracterizan por procesos de aprendizaje, habilidades, creencias, objetivos, estructuras y comportamientos particulares, se relacionan unos con otros a

ARBOR Vol. 188753 enero-febrero [2012] 63-73 ISSN: 0210-1963 
través de relaciones de mercado y de no mercado. Los tipos de redes y relaciones varían por tanto entre sectores en función de las características de su base de conocimiento, sus procesos de aprendizaje, las tecnologías básicas que utilizan, las características de la demanda y las complementariedades dinámicas. Por su parte, el conocimiento de los agentes está condicionado por las instituciones, incluyendo éstas tanto normas, como rutinas, hábitos, prácticas, reglas, leyes, estándares, etc., pudiendo ser algunas impuestas y otras consensuadas, unas obligatorias y otras optativas, unas formales y otras informales, unas nacionales o regionales y otras sectoriales. Por último, la demanda en un sistema sectorial, según Malerba (2004), está formada por consumidores individuales, pero también por empresas e instituciones, caracterizados cada uno de ellos por su conocimiento, sus procesos de aprendizaje, sus competencias y sus objetivos, y sujetos a factores sociales y a instituciones. No se trata por tanto de un agregado de compradores homogéneos, sino de agentes heterogéneos cuya interacción con los productores se conforma con las instituciones.

En la perspectiva sectorial, como se ha apuntado, tanto el conocimiento como los procesos de aprendizaje y las tecnologías son de gran importancia. Respecto a estas últimas, Malerba (2004) indica que, por lo general, para un sector industrial puede ser importante más de una tecnología, y lo mismo podríamos apuntar respecto a una tecnología dada (que puede ser relevante para más de un sector). No obstante, una matriz que pusiera en relación tecnologías y productos sí sería una herramienta útil para diferenciar un sector de otros. De lo anterior se deduce que en un sector determinado pueden existir empresas que deban dominar más de una tecnología, pero también que dentro de un sector dado, y particularmente entre las empresas de mayor tamaño, existen pocas diferencias entre sus perfiles de diversificación tecnológica.

En cuanto al conocimiento, la perspectiva sectorial se hace eco de la teoría evolucionista que apunta a diferencias significativas entre sectores y tecnologías en cuanto a su base de conocimiento y procesos de aprendizaje relacionados con la innovación. Así, el conocimiento difiere entre sectores en función de dominios o áreas (Malerba, 2004). Un primer dominio se refiere a las áreas científicas y tecnológicas que se encuentran en la base de la actividad innovadora de un sector. Un segundo se refiere a las aplicaciones, usuarios y a la demanda de productos del sector.

Continuando con Malerba (2004), existen otras dimensiones del conocimiento que son útiles para entender las actividades innovadoras en los sistemas sectoriales y que fueron postuladas por Nelson y Winter (1982) en su noción de regímenes tecnológicos. Una primera dimensión se refiere a la apropiabilidad ${ }^{1}$ o accesibilidad del conocimiento, entendida ésta como la oportunidad de obtener conocimiento externo a la empresa; e implica que a mayor accesibilidad existirá menor concentración industrial. El conocimiento accesible, además, puede ser interno o externo al sector; una mayor accesibilidad al primero implica menores posibilidades de apropiárselo (es más sencillo para los competidores la imitación de productos y procesos); la accesibilidad al conocimiento externo al sector se relaciona con el nivel y las fuentes de las oportunidades científicas y tecnológicas donde los factores del medio externo pueden afectar a las empresas a través del capital humano o del conocimiento científico y tecnológico desarrollado en organizaciones no empresariales.

En segundo lugar, las fuentes de oportunidades tecnológicas difieren notablemente entre sectores, como ya apuntaran Freeman (1982) y Rosenberg (1982), estando las oportunidades tecnológicas en ciertos sectores vinculadas a avances científicos en universidades, mientras que en otros estos avances son acometidos por la I+D desarrollada en empresas. Además, en otros sectores, como ya se ha señalado, las oportunidades para innovar provienen de proveedores (son incorporadas a través de la adquisición de bienes de equipo o de instrumentación) o de clientes. Más aún, que el conocimiento externo sea asimilado y aplicado en una industria concreta depende de su accesibilidad y de sus posibilidades para ser transferido: si éstas son altas, la entrada de innovaciones también será alta. Si, por el contrario, se requieren unas capacidades avanzadas para asimilarlo, la industria en cuestión será más proclive a configurarse en torno a grandes compañias previamente establecidas.

Además, y en tercer lugar, el conocimiento puede ser más o menos acumulativo en función de tres fuentes: la cognitiva, la capacidad organizativa y la retroalimentación desde el mercado. La fuente cognitiva se refiere a los procesos de aprendizaje y al conocimiento obtenido, que pueden 
limitar el desarrollo de nuevo conocimiento, pero también generar nuevo conocimiento. La capacidad organizativa es especifica de las empresas y genera conocimiento del tipo path-dependent, definiendo implícitamente lo que una empresa aprende y puede esperar obtener en el futuro. La retroalimentación desde el mercado se refiere a la oportunidad que representa para las empresas con innovaciones exitosas el reinvertir parte de esos beneficios en el desarrollo de nuevas innovaciones, configurando un círculo virtuoso. Y además, la acumulación puede darse a tres niveles de análisis diferentes: al nivel tecnológico, al de la empresa (cuando la apropiabilidad es alta), al sectorial (cuando la apropiabilidad es baja) o al nivel local (más factible cuando la apropiabilidad es baja y existen spill overs localizados en un área).

Según Malerba y Orsenigo (1993) hay evidencias que asocian ciertos tipos de regímenes de aprendizaje con comportamientos innovadores básicos. Así, las oportunidades tecnológicas se asocian más fácilmente con innovaciones radicales, mientras que la acumulación lo es con comportamientos innovadores incrementales, y la apropiabilidad o accesibilidad al conocimiento mediante la imitación.

De la propuesta de los SSI de Malerba (1999), que se centra en las dinámicas y las transformaciones de los sistemas sectoriales, destaca el énfasis en el conocimiento y en su estructura. Según él, la base de conocimiento difiere entre sectores y afecta a las actividades de innovación, a su organización y al comportamiento de las empresas.

Sobre las empresas (o unidad de análisis apropiada) la perspectiva SSI se interesa por sus procesos de aprendizaje, competencias, comportamiento y organización, poniendo el énfasis en la heterogeneidad de sus agentes y en la variedad de sus comportamientos y organización. Así, son las interdependencias y complementariedades las que suponen las verdaderas fronteras del SSI, que pueden estar al nivel input o de demanda, y pueden afectar a la innovación, a la distribución o a la producción. Enfatiza también el rol de las organizaciones no empresariales e instituciones y se interesa por las relaciones entre los agentes, sean éstas de mercado o no mercado.

El conocimiento, tanto tácito como codificado, juega un papel fundamental tanto para la innovación como para la producción. La base de conocimiento que está detrás de las actividades de las empresas deviene en altamente idiosincrático y no se difunde automáticamente ni sin costes, sino que debe ser absorbido por las empresas a través de las habilidades adquiridas.

Los sectores pueden tener multitud de vínculos y complementariedades que se extienden fuera de sus fronteras definidas en términos de la demanda o de sus tecnologías básicas. Los vínculos pueden ser estáticos, mientras que las complementariedades dinámicas comprenden las interdependencias y retroalimentaciones tanto al nivel de la demanda como al de la producción. Y ambos afectan a las estrategias, a la organización y rendimiento, la velocidad y dirección del cambio tecnológico, el tipo de competición y las redes entre los diferentes tipos de agentes.

En suma, esta perspectiva enfatiza la diversidad entre los agentes en términos de conocimiento y de capacidades, la importancia de la confianza, y el grado de interacciones informales y relaciones entre los agentes. E indica que, en contextos de incertidumbre y cambio, [...] las redes no surgen como consecuencia de la similitud de sus agentes, sino porque son diferentes. En este sentido las redes pueden integrar complementariedades en conocimiento, capacidades y especialización (Malerba, 1999, p. 17).

\section{Elementos desde la perspectiva del distrito INDUSTRIAL MARSHALLIANO}

Los distritos son sistemas productivos definidos geográficamente, caracterizados por un gran número de empresas que se ocupan de diversas fases y formas en la elaboración de un producto homogéneo. El concepto de distrito industrial se ha definido tradicionalmente como [...] una entidad socioeconómica que se caracteriza por la presencia activa de una comunidad de personas y una población de empresas en un área natural e históricamente delimitada (Becattini 1990, p. 39).

Entre los principales elementos de esta perspectiva cabe destacar que el distrito industrial asume la existencia de una población de empresas, generalmente pequeñas o muy pequeñas, que se especializan en una o más fases del proceso productivo. El distrito se caracteriza por ser un grupo de empresas trabajando conjuntamente, donde existe una

ARBOR Vol. 188753 enero-febrero [2012] 63-73 ISSN: 0210-1963 
división del trabajo más inter-empresa que intra-empresa. Esta concentración y especialización incrementa la tensión y necesidad de innovar, lo que a su vez refuerza la integración y los vínculos entre las empresas (Galetto, 2008).

Una de las caracteristicas del distrito industrial es que ha sido concebido como un todo social y económico [...] donde la industria principal y la comunidad local de familias e instituciones colectivas se solapan en el sentido que los valores, actitudes y decisiones de inversión de la comunidad son orientados por la presencia de la industria, y los factores industriales estratégicos están ligados a las relaciones socio-económicas desarrolladas en la comunidad (Bellandi, 1996, p. 2).

Esto implica que existe una estrecha interrelación entre las esferas social, política y económica, y que el funcionamiento de una de ellas, por ejemplo la económica, está determinado por el funcionamiento y la organización de las otras. Por tanto, el éxito económico no depende sólo del campo de lo económico, sino que son igualmente importantes los aspectos sociales e institucionales más amplios (Dei Ottati, 2006). A ello se suma, además, la existencia de un entramado institucional, público y privado, que ofrece servicios reales (Brusco, 1990).

Uno de los aspectos centrales a la teoría de los DIM es la existencia de economías externas (Marshall, 1997) a las empresas cuando se reúne en un mismo distrito un gran número de pequeños productores quienes, especializados en fases particulares del proceso productivo, son capaces de realizarlas con la máxima economía (eficiencia). Un distrito así configurado hará rentable hacer inversiones de capital a aquellas industrias subsidiarias que, bien les proveen de elementos especiales que aquellas requieren para cada fase concreta, bien se dedican a la recogida y distribución de los diferentes materiales que aquellas requieren, o bien se dedican a la recogida y distribución de los productos de su actividad (Becattini, 1979).

Esta concentración de empresas y actividades resulta en un mercado de trabajo formado, especializado y flexible, que hace que los trabajadores del distrito se caractericen por una elevada formación en la industria local y una alta especialización en las fases del proceso productivo (Galetto, 2008). Esto además promueve una circulación más rápida de las ideas (Becattini, 1979), al contar con una amplia población dedicada a pensar, reflexionar y experimentar, voluntaria o accidentalmente, sobre su trabajo, y que pertenece a una comunidad muy interconectada (atmósfera industrial en palabras de Marshall), lo que provoca que el conocimiento fluya por el distrito, potenciando la innovación y la productividad (eficiencia tecnológica y reducción de costes).

Así, la proximidad física, social y cognitiva entre los agentes del distrito hace más veloz y eficiente los procesos de difusión y absorción de las innovaciones, que en los DIM rara vez se dan mediante alianzas o cooperación directa entre las empresas (Boix, 2008). Y se debe a la combinación de varios fenómenos: 1) el intercambio informal de información en espacios públicos y domésticos; 2) la movilidad del factor trabajo; 3) la cadena de proveedores especializados articulados entorno a las demandas del integrador final; 4) las innovaciones en las fases de los proveedores; y 5) la imitación.

Lo anterior también propicia la competición o rivalidad que, siempre y cuando sus manifestaciones más agresivas están penalizadas por la comunidad, suponen un aliciente para asumir riesgos y comportamientos innovadores (BeIlandi, 1996).

Por otro lado, el mapa de los participantes en los distritos incluye como actores principales no sólo las empresas finales, sino los proveedores de los distintos productos y servicios intermedios y un amplio conjunto de instituciones (universidades, asociaciones empresariales, agentes de política industrial y otras instituciones locales o regionales). Así, las innovaciones tecnológicas y organizativas asumen en los DIM:

[...] las connotaciones de un proceso continuo, con una acumulación y una interdependencia de los efectos de un gran número de cambios tecnológicos, cada uno de los cuales es de pequeña entidad; y por consiguiente las connotaciones de un proceso innovador de tipo incremental (a la Rosenberg; [1983]), más bien que mediante grandes saltos (Schumpeter, [1946]) (Garofoli, 1989, p. 81).

de este modo, en los DIM el conocimiento práctico generado ya sea mediante

[...] el aprendizaje por la práctica o el aprendizaje mediante el uso, requiere una gran cantidad de actores interconecta- 
dos para satisfacer las demandas de intercambio continuo (aprendizaje mediante interacción). Por tanto, la mayoria de las innovaciones no se generan en departamentos de $I+D$, sino que se derivan de la creatividad espontánea (Boix, 2008, p. 7) o

por procesos de innovación desde abajo, lo que se llama creatividad industrial descentralizada (descentralización de las fuentes de nuevo conocimiento sobre los procesos de producción y sobre las actividades de uso de los productos, que escapa al control de la I+D). Esto favorece el cambio gradual entendido tanto como: a) variaciones limitadas en los mercados, en los factores o en la tecnología; o como b) el flujo de variaciones continuas que acompañan a los procesos de crecimiento sostenido (Bellandi, 1996).

La especialización entre diferentes actividades de la actividad industrial y otras relacionadas provoca diferentes núcleos de especialización en el saber hacer y de aproximaciones a la producción y a la innovación, cuya interacción favorece la aparición de combinaciones originales acerca de productos, procesos y mercados. En consecuencia, las pequeñas empresas están mejor capacitadas para abordar la especialización en mercados muy especializados de gama alta.

La accesibilidad interna en el DIM dificulta la apropiación, ya que favorece la imitación y el desarrollo de nuevas empresas por agentes ya imbricados en el entramado distrital, y por tanto favorece las innovaciones de tipo incremental.

Por tanto, y desde una perspectiva evolucionista, los DIM son:

[...] organismos económicos multicelulares inmersos en procesos de selección económica que se ven en la necesidad de cambiar sus rasgos a través de la innovación a fin de sobrevivir al proceso de creación destructiva (Boix, 2008, p. 7).

en el que las spin-off y la fragmentación de la cadena de producción serían facilitadores del proceso innovador.

Es posible que la estructura de DIM dificulte la adaptación a cambios de tipo más radical por su tendencia a la inercia, por el pequeño tamaño de las empresas y por su organización descentralizada que puede dificultar inver- siones estratégicas para abrir nuevos mercados, desarrollar innovaciones radicales basadas en nuevos conocimientos explícitos o fijar estándares. Pero también es cierto que su naturaleza mestiza puede favorecer el aprovechamiento de otra de las subunidades que componen el DIM, asegurando de este modo su supervivencia aunque cambie su orientación ${ }^{2}$, y que el papel de instituciones colectivas o la propia prestación de servicios reales en un DIM puede matizar mucho las dificultades derivadas de la descentralización (Bellandi, 1996). Así, el contacto cara a cara y la proximidad física y entre las empresas facilita la interacción y la transmisión de recursos y conocimiento, que resultaría difícil de conseguir con relaciones a larga distancia. En definitiva, el valor crítico de los distritos está más en los recursos sociales o relacionales que en las externalidades tangibles, las infraestructuras físicas o las de nuevo conocimiento.

\section{Síntesis y propuesta del enfoque del sistema DISTRITUAL DE INNOVACIÓN}

Vistas las principales aportaciones que desde ambas perspectivas integramos en nuestro enfoque, consideraremos ahora algunos de los aspectos de interés que podemos anticipar derivados de la utilización de los sistemas distrituales de innovación.

En un sistema distritual de innovación el tipo de redes que se establecen pueden favorecer la generación de diferentes tipos de innovaciones. Así, los contactos frecuentes que se establecen entre empresas pueden ser de gran utilidad para el desarrollo de innovaciones de tipo incremental, que requieren un conocimiento profundo en un área determinada. Este tipo de contactos, denominados de vínculos fuertes, sin embargo, no parecen ser los más propicios para el desarrollo de innovaciones de ruptura, no sólo porque estos contactos proporcionan información que, dado la frecuencia de los contactos, tiende a ser redundante, sino también porque esas mismas redes estables pueden mostrar sintomas de path dependency derivados de una percepción compartida de la realidad (group thinking). Es por ello que el participar en redes con vínculos débiles, puede ser una estrategia recomendable para aquellas empresas que desean propiciar la capacidad de cambiar de orientación (Fagerberg, 2003).

ARBOR Vol. 188753 enero-febrero [2012] 63-73 ISSN: 0210-1963

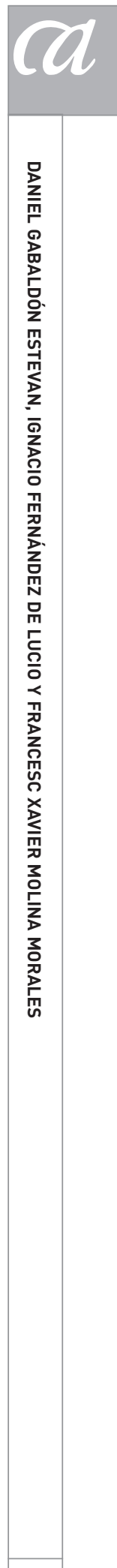


Cada innovación consiste en una nueva combinación de factores tales como ideas, capacidades, recursos, etc. Es por ello que el grado de apertura a nuevas ideas y soluciones es considerado esencial para la innovación, particularmente en sus primeras fases (Fagerberg, 2003). En consecuencia, cuanto mayor sea la variedad de dichos factores, mayor será la probabilidad de obtener innovaciones fruto de nuevas combinaciones de tales factores y más sofisticadas serán tales innovaciones.

Pudiera parecer que de lo anterior se derivase que los sistemas más amplios (naciones, regiones, etc.) tuviesen una posición de partida más ventajosa por disponer de más elementos constitutivos. No obstante, la necesidad ha hecho que sistemas de menor tamaño (como un sistema distritual de innovación) requieran la vigilancia constante de sus competidores y la necesidad de establecer sistemas de vigilancia y de interactuar con fuentes externas de conocimiento. Así, cuanto mayor es el número de empresas capaz de interactuar con fuentes externas de conocimiento, mayor es la presión sobre el resto de empresas para hacer lo propio, lo que impulsa la capacidad innovadora tanto de las empresas como de los sistemas a los que pertenecen (regionales, nacionales o distrituales). Lo anterior es particularmente relevante para las PYMEs, quienes deben compensar unos recursos internos limitados mediante una buena capacidad de interrelación con el mundo exterior. No obstante, la creciente complejidad de las bases de conocimiento necesarias para la innovación hace que incluso las grandes empresas dependan de manera creciente de fuentes externas en sus actividades de innovación (Fagerberg, 2003).

Kline y Rosenberg (1986) subrayaron el hecho de que la innovación no debe ser entendida como algo bien definido y homogéneo que aparece o esté disponible en un momento preciso, sino que, en la mayoría de los casos, las innovaciones atraviesan cambios drásticos en sus vidas que muy a menudo suponen también cambios drásticos en su significación económica. De hecho, muchas mejoras posteriores a la primera introducción de una invención tienen consecuencias económicas mucho más importantes que la inicial. En esta línea, Nelson y Rosenberg (1993) indicaron que la empresa innovadora schumpeteriana, aquella que primero trae un producto al mercado, no es, generalmente, aquella que en último término captura la mayor parte de las rentas asociadas con la innovación. Una innovación exitosa no es tanto una cuestión de invención como una cuestión de diseño. La actividad innovadora dependerá por tanto de las interacciones del conjunto de instituciones que conforman el sistema distritual de innovación.

La visión sistémica presenta una serie de condiciones que merecen tenerse en cuenta. En primer lugar, implica que los agentes y las instituciones son considerados en función de su contribución a la innovación. Un aspecto fundamental de la mejora del proceso de innovación implica revisar y rediseñar los enlaces entre las partes del sistema. Esta visión asume ciertas imperfecciones en el mercado de las innovaciones, que requieren una intervención política sobre el mismo. Las competencias son distribuidas irregularmente entre las empresas; las buenas prácticas de innovación no son difundidas de forma inmediata entre las empresas y los fallos de mercado pueden incluir fallos de las instituciones para coordinar, conectar y satisfacer las necesidades del sistema, etc. Por otro lado, se asume que el entramado institucional difiere de un territorio a otro y que ciertas fases del proceso son más idóneas para las empresas en un territorio o país específico. En definitiva, los sistemas de innovación han de servir para asegurar el flujo de información a través de interfaces entre empresas, centros de investigación, emprendedores, inversores de todo tipo, consultorías, agencias de patentes, instituciones locales y otros intermediarios (Lundvall y Borrás, 2005).

De manera genérica, desde la perspectiva del sistema distritual de innovación la innovación se concibe como un proceso interactivo en el que una multitud de agentes de diferente naturaleza (clientes, empresas y otras organizaciones como universidades, centros de investigación, administraciones públicas, instituciones financieras...) participan en el proceso de innovación. Se subraya así la importancia de las redes que conectan los diferentes elementos del sistema (ya que las empresas no innovan de manera aislada sino en interacción con otras empresas, así como con otras organizaciones y elementos del sistema), y la importancia de los mecanismos de intercambio y retroalimentación de la información y del stock de conocimientos.

EI SSI se distingue por utilizar como ámbito de delimitación de su análisis la noción de sector industrial (definido éste en términos de producto) $y_{1}$ en consecuencia, le son de interés aquellas empresas, agentes e instituciones que se vinculan con la actividad sectorial independientemente 
de su ubicación. En un sistema distritual de innovación, sin minusvalorar la relevancia de agentes externos al distrito, el énfasis radica en los tipos de relaciones que se generan en función de encontrarse éstos en un mismo ámbito territorial. En cierto modo, la propuesta de Malerba deja varias puertas abiertas que facilitan su conexión con la propuesta que aquí realizamos. Por una parte indica que cuando se produce una

[...] alta acumulación de conocimiento en localizaciones espaciales concretas, es más probable que se asocie con condiciones de baja apropiación y spill overs de conocimiento espacialmente localizados (Malerba, 1999, p. 9).

lo que coincide con el ámbito distritual de nuestro planteamiento. Por otro lado, indica que el análisis de los agentes que intervienen en un SSI puede ser diferente (superior o inferior) al de sector (Malerba, 1999, p. 15). Y más adelante precisa que en algunos sectores, las redes pueden constituir sistemas locales (regionales) de innovación y producción (Malerba, 1999, p. 17), para reconocer finalmente que

[...] una tradición próxima a los sistemas sectoriales son los estudios de sistemas regionales o locales: de hecho a menudo un sistema local coincide con un sector (vean, por ejemplo, los estudios sobre distritos industriales y de la industria de la maquinaria) (Malerba, 1999, p. 30).

Por su parte, las principales corrientes que han desarrollado las teorías sobre el distrito industrial lo hicieron a partir del rechazo de la unidad de análisis sectorial, por considerar que no era la más adecuada para explicar los principales fenómenos que afectan al desarrollo de los sistemas productivos locales. En palabras de Becattini (1979), de la lectura de Marshall se extrae que el distrito industrial es la unidad indivisible, el átomo del que debe partir la investigación industrial. Además, la localización espacial y la naturaleza multisectorial que caracteriza a los DIM le otorgan una estabilidad mayor, frente a situaciones de cambios intensos, de lo que la industria, el sector o la tecnología pudieran ofrecer. De hecho, cuantas más transiciones experimente el DIM, más refuerza su identidad (Becattini, 1979).

De nuevo desde la perspectiva sistémica se ha enfatizado cómo la interconexión, que facilita interacciones y sinergias, entre empresas que no disponen de grandes recursos puede suplir la falta de éstos. Así, Hobday (1991) describe los principales mecanismos que ayudan a estas empresas. En primer lugar, grupos de pequeñas empresas pueden mantener tecnologías punta apoyándose en otras organizaciones de la red. En segundo lugar, la acumulación de competencias y el aprendizaje colectivo se produce dentro de la red, beneficiando a todos los participantes. En tercer lugar, la red promueve el flujo de individuos clave entre empresas. En cuarto lugar, las competencias pueden combinarse y recombinarse para superar los cuellos de botella. Además, en quinto lugar, el tiempo y los costes de innovar pueden reducirse. En sexto lugar, la red proporciona la entrada a la industria a nuevas empresas innovadoras. Por último, las empresas operan en la red con gran flexibilidad y costes reducidos. Estas caracteristicas coinciden con las denominadas ventajas competitivas de las economías de distrito, esto es, derivadas de un conjunto fuertemente interconectado de economías externas a las empresas, pero internas al distrito (Dei Ottati, 2006, p. 74) y que se sintetizan en: 1) eficiencia en el empleo de los recursos, particularmente el trabajo e inputs intermedios; y 2) la innovación como resultado de la acumulación de capital humano especializado, de la dinámica competitiva, y de la rápida difusión de la información.

\section{Conclusiones}

En el contexto de este trabajo entendemos la noción de Sistema Distritual de Innovación como un sistema de relaciones donde se generan externalidades para las empresas que facilitan sus procesos de innovación y que se producen en el seno de un distrito industrial, entidad de carácter social que si bien se vincula a un territorio, generalmente no se ajusta a límites administrativos concretos. Desde esta perspectiva, un SDI requiere que la unidad de análisis se amplíe para abarcar no sólo las empresas e instituciones que configuran el distrito industrial, sino que incluya aquellos elementos de su SSI, concebido tanto como sistema tecnológico y como de producto, con los que interactúa, se sitúen éstos dentro del mismo sistema regional de innovación o fuera de él.

Por lo tanto, el elemento definitorio de qué agentes e instituciones entran dentro de la unidad de análisis del SDI será el que se derive de su contribución a la in-

ARBOR Vol. 188753 enero-febrero [2012] 63-73 ISSN: 0210-1963 
novación del distrito en consideración. De esta manera pretendemos salvar las limitaciones de un acotamiento de la actividad industrial en función de un producto, que nos obligaría a un tratamiento en segundo plano de otras actividades de gran relevancia para la comunidad donde se inserta el distrito (Fernández et al., 2005; Tortajada Esparza et al., 2008). Tratamos de salvar también las limitaciones de una clasificación de la actividad industrial basada sólo en la tecnología, ya que la contribución de una tecnología no deja de ser algo relativamente coyuntural al desarrollo de cualquier industria (Becattini, 1979). $Y$, más específicamente para nuestro enfoque, tampoco un cambio tecnológico considerable desvirtuaría nuestra unidad de análisis (Ybarra, 2007), ni todas las actividades industriales que, en un momento dado, utilicen las mismas tecnologías del SDI bajo estudio, tienen por qué influir en su desarrollo. Por otro lado, tal y como apuntara Becattini (1979), el sentimiento de pertenencia a una industria (como elemento de psicología colectiva), que está en la base de la definición de DIM, puede sernos útil a la hora de acotar nuestra unidad de análisis si somos capaces de trascender la comunidad primigenia local en la que surge y abarcamos las redes sectoriales en las que se estructura (Molina-Morales, 2008), y si mantenemos que el objetivo de tal acotación es el estudio a posteriori de una realidad productiva.

Queda claro, por tanto, que lo que proponemos no es una visión radicalmente novedosa para analizar la innovación en aglomeraciones industriales sino, muy al contrario, la síntesis de dos perspectivas, la de los sistemas de innovación y los distritos industriales, que vienen analizando el desarrollo industrial con herramientas y niveles de análisis

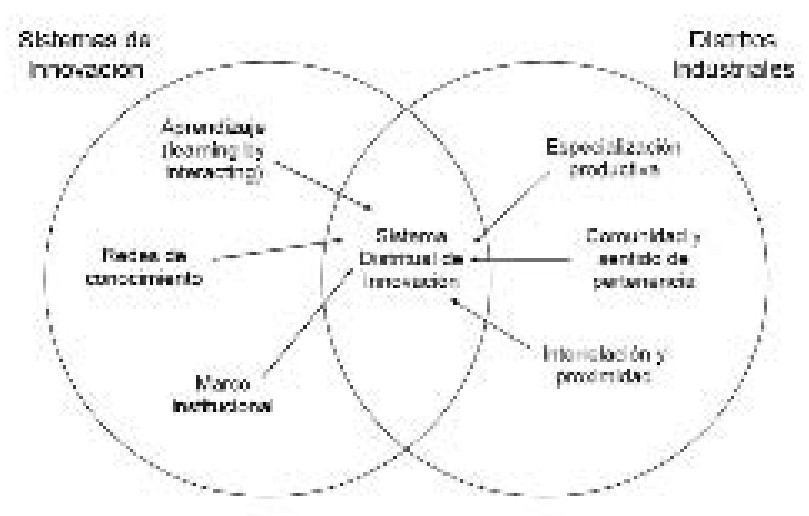

Figura 1. La perspectiva Sistema Distritual de Innovación

diferentes pero complementarios. Los componentes que constituyen el foco del análisis del sistema distritual de innovación, a partir de la combinación de las perspectivas citadas, se ilustran en la Figura 1. Desde la primera se resaltan los procesos de aprendizaje, las redes de conocimiento y el marco institucional, mientras que desde la segunda se subrayan la especialización productiva, la importancia de los lazos comunitarios y la interrelación y proximidad. Estas perspectivas estarian insertas en las disciplinas de la sociología económica, junto a la economía evolucionista, la economía regional y la geografía económica. De la exposición se deriva la necesidad de un término y un marco de análisis bajo el que se agrupen aquellas investigaciones que tratan de analizar la innovación en un entorno industrial que adopta la forma de distrito industrial haciendo uso de un análisis sistémico basado en el aprendizaje a través de las interacciones.

\section{NOTAS}

1 Se entiende por apropiabilidad la capacidad de proteger las innovaciones de la imitación, permitiendo la explotación y la obtención de beneficios derivados (Levin et al., citado en Malerba, 2004, p. 21).

Recibido: 1 de noviembre de 2010 Aceptado: 10 de abril de 2011
2 Para un ejemplo ilustrativo ver el análisis del sector del juguete en Ibi por Ybarra (2007).

\section{BIBLIOGRAFÍA}

Asheim, B. T. y Isaksen, A. (2001), "Los sistemas regionales de innovación, las PYMEs y la política de innovación", en Olazaran, M. y Gómez, M. (coords.), Sistemas regionales de innovación, Bilbao: Universidad del País Vasco, pp. 93-114.

Becattini, G. (1979), "Dal Settore Industriale al Distretto Industriale. Alcune 
considerazioni sull'unitá di indagine in economia industriale", Revista di Economia e Politica Industriale, 1: 7-14.

Becattini, G. (1990), "The Marshallian industrial district as a socio-economic notion", en Pyke, F.; Becattini, G. y Sengenberger, W. (eds.), Industrial districts and inter-firm co-operation in Italy, Ginebra: International Institute for Labour Studies, pp. 37-51.

Bellandi, M. (1996), "Innovation and change in the Marshallian Industrial District", European Planning Studies, 4 (3): 357-369.

Boix, R. (2008), "Industrial districts, innovation and I-district effect: territory or industrial specialization?", Working Papers wpdea0807, Barcelona: Departament d'Economia Aplicada. Universitat Autònoma de Barcelona.

Brusco, S. (1990), "The idea of the industrial district: its genesis", en Pyke, F.; Becattini, G. y Sengenberger, W. (comps.), Industrial districts and interfirm co-operation in Italy, Ginebra: International Institute for Labour Studies.

Dei Ottati, G. (2006), "El 'efecto distrito': algunos aspectos conceptuales de sus ventajas competitivas", Economía Industrial (359): 73-79.

Dosi, G.; Pavitt, K. y Soete, L. (1990), The Economics of Technical Change and International Trade, Brighton: Harvester Wheatsheaf.

Edquist, C. (ed.) (1997), Systems of innovation; Technologies, Institutions, and Organizations, London: Pinter.

Fagerberg, J. (2003), "Innovation: A Guide to the Literature", Working Papers on Innovation Studies 20031012, Oslo: Centre for Technology, Innovation and Culture, University of Oslo.

Fernández, I.; Gabaldón, D. y Gómez, C. (2005), La innovación en el sector de pavimentos y revestimientos cerá- micos de la Comunidad Valenciana, Valencia: Alto Consejo Consultivo en I+D de la Presidencia de la Generalitat Valenciana.

Freeman, C. (1987), Technology and Economic Performance: Lessons from Japan, Londres: Pinter.

Galetto, V. (2008), "Distrito industrial e innovación", en Soler, V. (coord.), Los distritos industriales, El Ejido (Almería): Cajamar Caja Rural Intermediterránea, pp. 117-137.

Garofoli, G. (1989), "Modelli Locali di Sviluppo: I Sistemi di Piccola Impresa", en Becattini, G. (ed.), Modelli Locali di Sviluppo, Bolonia: II Mulino, pp. 7590.

Hobday, M. (1991), Dynamic networks, technology diffusion and complementary assets: Explaining U.S. decline in semiconductors, DRC Discussion Papers, 78. Falmer (U.K): Science Policy Research Unit, University of Sussex.

Kline, S. J. y Rosenberg, N. (1986), An overview of innovation, Washington, DC: National Academy Press.

Lundvall, B. A. (1992), National systems of innovation: Towards a theory of innovation and interactive learning, Londres: Printer.

Lundvall, B. A. y Borrás, S. (2005), "Science, Technology, and Innovation Policy", en Fagerberg, J.; Mowery, D. C. y Nelson, R. R. (eds.), The Oxford Handbook of Innovation, Nueva York: Oxford University Press, pp. 599-631.

Malerba, F. (1999), "Sectoral systems of innovation and production", DRUID Conference on: National Innovation Systems, Industrial Dynamics and Innovation Policy, Rebild. Disponible en: http://www.druid.dk/uploads/tx_ picturedb/ds1999-69.pdf. [Consulta: 12 de abril de 2007].

Malerba, F. (2004), "Sectoral Systems of Innovation: Basic Concepts", en Malerba, F. (ed.), Sectoral Systems of Inno- vation: Concepts, Issues and Analyses of Six Major Sectors in Europe, Reino Unido: Cambridge University Press, pp. 9-41.

Malerba, F. y Orsenigo, L. (1993), "Technological regimes and firm behavior", Industrial and corporate change (2): 45-74.

Marshall, A. (1997), Principles of economics, New York: Prometheus books.

Molina-Morales, F. X. (2008), "Los distritos industriales en la Europa mediterránea: las diferencias entre Italia y España", en Soler, V. (coord.), Los distritos industriales, El Ejido (Almeria): Cajamar Caja Rural Intermediterránea, pp. 183-201.

Nelson, R. y Rosenberg, N. (1993), "Technical Innovation and National Systems", en Nelson, R. (ed.), National Innovation Systems. Nueva York: Oxford University Press, pp. 3-21.

Nelson, R. R. y Winter, S. G. (1982), An Evolutionary Theory Of Economic Change, Cambridge: Belknap Press.

Oinas, P. y Malecki, E. J. (2002), "The Evolution of Technologies in Time and Space: from National and Regional to Spatial Innovation Systems", International Regional Science Review, 25 (1): 102-131.

Rosenberg, N. (1982), Inside the Black Box: Technology and Economics, Cambridge: Cambridge University Press

Tortajada Esparza, E.; Gabaldón Estevan, D. y Fernández de Lucio, I. (2008), "La evolución tecnológica del distrito cerámico de Castellón: la contribución de la industria de fritas, colores y esmaltes", Boletín de la Sociedad Española de Cerámica y Vidrio, 47 (1): 57-80.

Ybarra, J. A. (2007), La innovación en el sector del juguete de la Comunidad Valenciana, Valencia: Fundación Premios Rey Jaime I. Presidencia de la Generalitat Valenciana. 